\title{
Evaluation and validation of suitable reference genes for reverse transcription-quantitative polymerase chain reaction studies in cholangiocarcinoma patients and cell lines
}

\author{
JINGHUI YANG ${ }^{1}$, QIWEI YANG ${ }^{2}$, SHAN YU ${ }^{3}$ and XUEWEN ZHANG ${ }^{1}$ \\ ${ }^{1}$ Department of Hepatopancreatobiliary Surgery, China-Japan Union Hospital of Jilin University, Changchun, Jilin 130033; \\ ${ }^{2}$ Central Laboratory, Second Hospital of Jilin University, Changchun, Jilin 130041; ${ }^{3}$ Department of Neurology, \\ China-Japan Union Hospital of Jilin University, Changchun, Jilin 130033, P.R. China
}

Received December 14, 2014; Accepted January 29, 2016

DOI: $10.3892 / \mathrm{ol} .2016 .4232$

\begin{abstract}
Reverse transcription-quantitative polymerase chain reaction (RT-qPCR) has become a frequently used method in gene expression studies. The relative quantification method is an important and common method for the evaluation of RT-qPCR data. One of the key requirements of this method is to identify an applicable internal reference gene. However, to the best of our knowledge, no suitable reference genes have been identified for the genetic analysis of cholangiocarcinoma (CCA) in humans and cell lines. In the present study, screening was conducted using 12 common reference genes, which were selected in order to provide an experimental basis for the study of the gene expression in CCA patients and cell lines. Tumor samples and adjacent non-neoplastic tissues from 20 patients with CCA were obtained for the present study. The stability and applicability of the 12 reference gene candidates were validated using GeNorm, NormFinder and BestKeeper software. Based on a comparison of the results, the following reference genes are recommended for various tissue groups: Total sample group, ribosomal protein L29; CCA tissue group, TATA-box binding protein; CCA cell line and tissue group, actin- $\beta$; CCA cell line group, $18 \mathrm{~S}$ ribosomal RNA; CCA tissue and adjacent non-neoplastic tissue group, 5'-aminolevulinate synthase 1; and adjacent non-neoplastic tissue group, hypoxanthine phosphoribosyltransferase 1 . The results of the search
\end{abstract}

Correspondence to: Professor Xuewen Zhang, Department of Hepatopancreatobiliary Surgery, China-Japan Union Hospital of Jilin University, 126 Xiantai Street, Changchun, Jilin 130033, P.R. China

E-mail: xuewenzhang01@126.com

Dr Shan Yu, Department of Neurology, China-Japan Union Hospital of Jilin University, 126 Xiantai Street, Changchun, Jilin 130033, P.R. China

E-mail: adashan521@sina.com

Key words: cholangiocarcinoma, reverse transcription-quantitative polymerase chain reaction, reference gene, GeNorm, NormFinder, BestKeeper also clearly reveal that a systematic study regarding the selection of suitable reference genes for studying the target gene profiling in CCA tissues and cell lines has not been previously published. The present study may provide useful information for future studies that examine the gene expression of CCA for choosing suitable reference genes.

\section{Introduction}

Cholangiocarcinoma (CCA) is an uncommon tumor that may originate from anywhere in the biliary epithelium (1). In America, the incidence of this disease is 1.4-1.7 per 100,000 individuals $(1,2)$, whilst Chinese patients with CCA account for $>55 \%$ of cases worldwide (3). Complete tumor resection is considered to be the most efficient therapy for CCA; however, only $10 \%$ of patients are regarded as candidates for surgical resection as CCA may be highly malignant and progress rapidly, making surgical resection no longer an option for the majority of patients upon diagnosis (1). Long-term survival remains poor in patients that are not candidates for surgical resection, and the 5-year survival rate is $\sim 5 \%$ (4).

Reverse transcription-quantitative polymerase chain reaction (RT-qPCR) is regarded, at present, as the gold standard for efficient and sensitive analysis of gene expression (5-7). In order to produce reliable data, however, gene expression levels must be normalized using two or more reference genes (8-10). Ideal reference genes are those which are stable, unregulated and invariable under the conditions of the experiment. Thus, it is important to conduct validation experiments to assess reference gene expression stability for each target tissue and disease $(11,12)$. To the best of our knowledge, studies on the selection of suitable reference genes for use in target gene profiling in CCA tissues and cell lines have not been previously published.

The aim of the present study was to identify the most suitable reference gene or set of genes for target gene profiling of CCA. The stability of 12 common reference genes were validated in CCA tissues and paired normal tissues from 20 patients with CCA. In addition, the stability of the reference genes was validated in one CCA cell line. Three common programs, GeNorm (8) NormFinder (13) and BestKeeper (14), 
were employed for analysis of the reference genes. In order to determine the validity of the reference genes, a target gene, KRAS, which is closely associated with CCA (15), was used as a normalizer. The present study provides useful information regarding the selection of a suitable reference gene to utilize in future gene expression studies in CCA tissues and cell lines.

\section{Materials and methods}

CCA samples. A total of 20 tissue samples were obtained from patients that were treated for CCA by resection surgery between January 2009 and December 2013 at China-Japan Union Hospital of Jilin University (Changchun, China). Written informed consent was obtained from the patients for the use of their tissues in the present study. Paired normal samples were collected from tissues adjacent to the tumor. All samples were snap-frozen in liquid nitrogen immediately following excision and stored at $-80^{\circ} \mathrm{C}$ until required. All tumors were histopathologically diagnosed and staged according to the American Joint Committee on Cancer (16). The characteristics of the patients are summarized in Table I. The study protocol was approved by the Ethics Committee of the China-Japan Union Hospital.

Cell lines. The human CCA cell line QBC939 was purchased from the Cell Bank of Type Culture Collection of Chinese Academy of Sciences (Shanghai, China) and was cultured in RPMI-1640 (Invitrogen ${ }^{\circledR}$; Thermo Fisher Scientific, Inc., Waltham, MA, USA) supplemented with $10 \%$ fetal bovine serum and $0.02 \mathrm{mg} / \mathrm{ml}$ kanamycin (both Gibco ${ }^{\circledR}$; Thermo Fisher Scientific, Inc.).

$R N A$ extraction and $R T$. Tissue samples and the cell line were homogenized in Trizol ${ }^{\circledR}$ Reagent (Invitrogen ${ }^{\circledR}$; Thermo Fisher Scientific, Inc.) and purified with the RNeasy ${ }^{\circledR}$ Mini kit (Qiagen, Inc., Valencia, CA, USA). DNase I (Qiagen, Inc., Valencia, CA, USA) was used to eliminate genomic DNA contamination. Concentrations and quality of the isolated RNA were measured using a Synergy ${ }^{\mathrm{TM}}$ HT microplate reader (Bio-Tek Instruments, Inc., Winooski, VT, USA) at a $\mathrm{A}_{260} / \mathrm{A}_{280}$ ratio. The quality standard of the RNA samples was 1.9-2.2. The integrity of RNA samples was determined using electrophoresis on a $1 \%$ agarose gel (Invitrogen; Thermo Fisher Scientific, Inc.). RT was performed using an All-in-One ${ }^{\mathrm{TM}}$ First-Strand complementary (c)DNA Synthesis kit (GeneCopoeia, Inc., Rockville, MD, USA) in a total volume of $25 \mu 1$, following the manufacturer's protocol.

$q P C R$. Primer pairs of 12 putative reference genes were designed using Primer Premier version 5.0 (Premier Biosoft International, Palo Alto, CA, USA) and were synthesized by Sangon Biotech Co., Ltd. (Shanghai, China) (Table II). A LightCycler ${ }^{\circledR} 480$ Real-Time PCR System (Roche Diagnostics GmbH, Mannheim, Germany) was used for qPCR. Reactions were performed with All-in-One ${ }^{\mathrm{TM}}$ qPCR Mix (GeneCopoeia, Inc.), according to the manufacturer's protocol. All samples were run in triplicate on 96-well plates. The total PCR volume was $20 \mu \mathrm{l}$, comprising $2 \mu \mathrm{l}$ cDNA. The following cycling conditions were used: $55^{\circ} \mathrm{C}$ for $5 \mathrm{~min} ; 95^{\circ} \mathrm{C}$ for $5 \mathrm{~min}$,
Table I. Clinicopathological characteristics of 20 patients with cholangiocarcinoma.

\begin{tabular}{lc}
\hline Characteristic & Value \\
\hline Age, years; mean \pm standard deviation & $54 \pm 16.7$ \\
Gender, $\mathrm{n}$ & 12 \\
Male & 8 \\
Female & \\
Histopathological type, $\mathrm{n}$ & 20 \\
Adenocarcinoma & 0 \\
Squamous cell carcinoma & \\
TNM stage ${ }^{\mathrm{a}}, \mathrm{n}$ & 4 \\
T1aN0M0 & 6 \\
TlbN0M0 & 6 \\
T2aN1M0 & 4 \\
T2bN1M0 & \\
\hline
\end{tabular}

${ }^{\mathrm{a} A s}$ characterized by the American Joint Committee on Cancer (16). $\mathrm{T}$, tumor; $\mathrm{N}$, node; $\mathrm{M}$, metastasis.

then 40 cycles of $95^{\circ} \mathrm{C}$ for $20 \mathrm{sec}, 55^{\circ} \mathrm{C}$ for $20 \mathrm{sec}$ and $72^{\circ} \mathrm{C}$ for $4 \mathrm{~min}$. The cycle was followed by melting curve analysis, and baseline and cycle threshold values (Cq values) were automatically determined for all samples using Roche LightCycler 480 software (version 1.5). A standard curve was constructed for each primer pair to determine product specificity. $\mathrm{Cq}$ values were identified using quantitative comparison of the amplification of the candidate genes. $\mathrm{Cq}$ values were calculated to relative quantities for data analysis according to the PCR efficiencies of the candidate genes using the following equation (17): $\mathrm{Q}=2^{-\Delta \mathrm{Cq}}$.

PCR efficiency. A random pool of cDNA was selected from the 20 patient samples and was 2-fold serially diluted (range, 1:1-1:100,000). The PCR efficiencies were calculated using the slopes of the calibration curves and the following formula: $\mathrm{E}=10^{-1 / \text { slope }}$ (18). PCR efficiencies are presented in Table II.

Evaluation of reference gene stability. Statistical analyses were conducted with GraphPad Prism software (version 5.0; GraphPad Software, Inc., La Jolla, CA, USA) and SPSS 16.0 (SPSS Inc., Chicago, IL, USA). In order to evaluate the differential expression of target genes, one-way analysis of variance with the Dunnett's test was used. $\mathrm{P}<0.05$ was considered to indicate a statistically significant difference.

The samples were divided into the following six groups: CCA tissue; adjacent non-neoplastic tissue; CCA cell line; matched pairs of adjacent non-neoplastic and CCA tissues; CCA tissue and cell line group; and total samples. Subsequently, to improve the evaluation of the stability of the reference genes, three frequently used programs were selected: GeNorm (genorm.cmgg.be/), NormFinder (moma.dk/normfinder-software) and BestKeeper (gene-quantification.de/bestkeeper.html). GeNorm has been designed to establish reference genes for RT-qPCR and analyzes and determines the M-value of reference genes. The 
Table II. Primer sequences, product size and PCR efficiency of 12 reference genes and KRAS, a target gene.

\begin{tabular}{|c|c|c|c|c|}
\hline \multirow[b]{2}{*}{ Gene } & \multicolumn{2}{|c|}{ Primer sequences, 5'-3' } & \multirow{2}{*}{$\begin{array}{l}\text { Product } \\
\text { size, bp }\end{array}$} & \multirow{2}{*}{$\begin{array}{c}\text { PCR } \\
\text { efficiency }\end{array}$} \\
\hline & Forward & Reverse & & \\
\hline 18SrRNA & CGGCTACCACATCCAAGGAA & GCTGGAATTACCGCGGCT & 186 & 2.12 \\
\hline GAPDH & GACAGTCAGCCGCATCTTCT & TTAAAAGCAGCCCTGGTGAC & 127 & 1.98 \\
\hline $\mathrm{B} 2 \mathrm{M}$ & AGCGTACTCCAAAGATTCAGGTT & ATGATGCTGCTTACATGTCTCGT & 206 & 1.96 \\
\hline ACTB & AGAAAATCTGGCACCACACC & TAGCACAGCCTGGATAGCAA & 173 & 1.98 \\
\hline ALAS1 & GGCAGCACAGATGAATCAGA & CCTCCATCGGTTTTCACACT & 150 & 2.01 \\
\hline GUSB & AGCCAGTTCCTCATCAATGG & GGTAGTGGCTGGTACGGAAA & 160 & 1.88 \\
\hline HPRT1 & GACCAGTCAACAGGGGACAT & CCTGACCAAGGAAAGCAAAG & 132 & 1.96 \\
\hline PBGD & AGTGTGGTGGGAACCAGC & CAGGATGATGGCACTGAACTC & 144 & 2.11 \\
\hline PPIA & AGACAAGGTCCCAAAGAC & ACCACCCTGACACATAAA & 118 & 1.96 \\
\hline PUM1 & CAGGCTGCCTACCAACTCAT & GTTCCCGAACCATCTCATTC & 211 & 2.01 \\
\hline RPL29 & GGCGTTGTTGACCCTATTTC & GTGTGTGGTGTGGTTCTTGG & 120 & 2.00 \\
\hline ТВР & TGCACAGGAGCCAAGAGTGAA & CACATCACAGCTCCCCACCA & 132 & 2.16 \\
\hline KRAS & ACCGGAAGCAGGTGGTCAT & CTTGGTGTTGTTGATGGCAAA & 146 & 1.97 \\
\hline
\end{tabular}

PCR, polymerase chain reaction; 18SrRNA, 18S ribosomal RNA; GAPDH, glyceraldehyde 3-phosphate dehydrogenase; B2M, $\beta$-2-microglobulin; ACTB, actin- $\beta$; ALAS1, 5 '-aminolevulinate synthase 1 ; GUSB, glucuronidase- $\beta$; HPRT1, hypoxanthine phosphoribosyltransferase 1; PBGD, porphobilinogen deaminase; PPIA, peptidylprolyl isomerase A; PUM1, pumilio RNA binding family member 1; RPL29, ribosomal protein L29; TBP, TATA-box binding protein; KRAS, Kirsten rat sarcoma viral oncogene homolog.

M-value is the stability of reference gene expression. The higher the M-value, the poorer the stability of the reference gene; the default value suggested by GeNorm is $M=1.5$. If the M-value is $>1.5$, it is not suitable to be used as a stable and reliable reference gene (8).

GeNorm software may also be used to analyze the pairwise variation value of the normalization factor (V), which has a default value of 0.15 . Pairwise variation $\left(V_{n / n+1}\right)$ was calculated between the two sequential normalization factors $\left(\mathrm{NF}_{\mathrm{n}}\right.$ and $\left.\mathrm{NF}_{\mathrm{n}+1}\right)$ for all samples to determine the optimal number of reference genes for reliable normalization. The value of $\mathrm{V}_{\mathrm{n}} / \mathrm{V}_{\mathrm{n}+1}$ may be used to determine if adding a new reference gene may greatly affect the normalization of the Fact-value. If the value of $\mathrm{V}_{\mathrm{n}} / \mathrm{V}_{\mathrm{n}+1}$ is $>0.15, \mathrm{n}+1$ reference genes are required as internal controls. If $\mathrm{V}_{\mathrm{n}} / \mathrm{V}_{\mathrm{n}+1}$ is $<0.15$, then it is not required to use new reference genes. NormFinder software has also been designed to identify the optimal reference gene among a set of candidate genes and it has a similar operation principle to GeNorm. It analyzes expression data, ranks a set of candidate normalization genes according to their expression stability and considers the gene with the minimum expression data as the most stable gene. This software may also be used to compare the stability of inter- and intra-group reference genes. BestKeeper evaluates candidate reference gene stability based on the standard deviation (SD) and correlation coefficient (r-value). If the SD is $>1$, the gene is not suitable to be used as a stable and reliable reference gene, and the higher the r-value, the more stable the reference gene.

Target gene relative expression analysis. KRAS is a proto-oncogene and is important in CCA development (15). The present study evaluated KRAS as a target gene, and the primer sequence is shown in Table II. The analysis of the
20 paired CCA tissue samples for target gene relative expression was calculated according to the $2^{-\Delta \Delta \mathrm{Cq}}$ method (19) using various candidate reference genes as the standard.

\section{Results}

RNA quality. In order to avoid erroneous results, only high-quality RNA samples were used in the present study. Total RNA samples were assessed for concentration, purity and integrity. The mean $\mathrm{A}_{260} / \mathrm{A}_{280}$ ratio of the RNA samples was $2.01 \pm 0.045$. The integrity of RNA samples was characterized by the $28 \mathrm{~S} / 18 \mathrm{~S}$ ratio, which was $>1.5$, on $1 \%$ agarose gels.

The sequences of primers, corresponding length of the amplified products and the PCR amplification efficiency are shown in Table II. The present study used two methods to verify the specificity of the primers. Firstly, qPCR amplification products were detected using $1 \%$ agarose gel electrophoresis (Fig. 1). Gel imaging demonstrated that the size of the amplified fragments of the reference genes was as expected; the bands were clear and there were no primer dimers or nonspecific banding. In addition, by observing the melting curve of each amplified gene fragment using qPCR, it was demonstrated that all curves had single signal peaks. For the candidate reference genes and the target gene, the amplification efficiency of the standard curve was 1.88-2.16, and all r-values were $>0.98$.

Gene expression. The expression level of the candidate reference genes was determined by the $\mathrm{Cq}$ value, which is inversely proportional to the expression level of a gene; the greater the $\mathrm{Cq}$ value, the smaller the expression quantity, and vice versa. As shown in Fig. 2, the Cq value of all the samples ranged from $24.52 \pm 4.52$ to $35.74 \pm 3.67$. In all the groups, glyceraldehyde 3-phosphate dehydrogenase (GAPDH) had the 


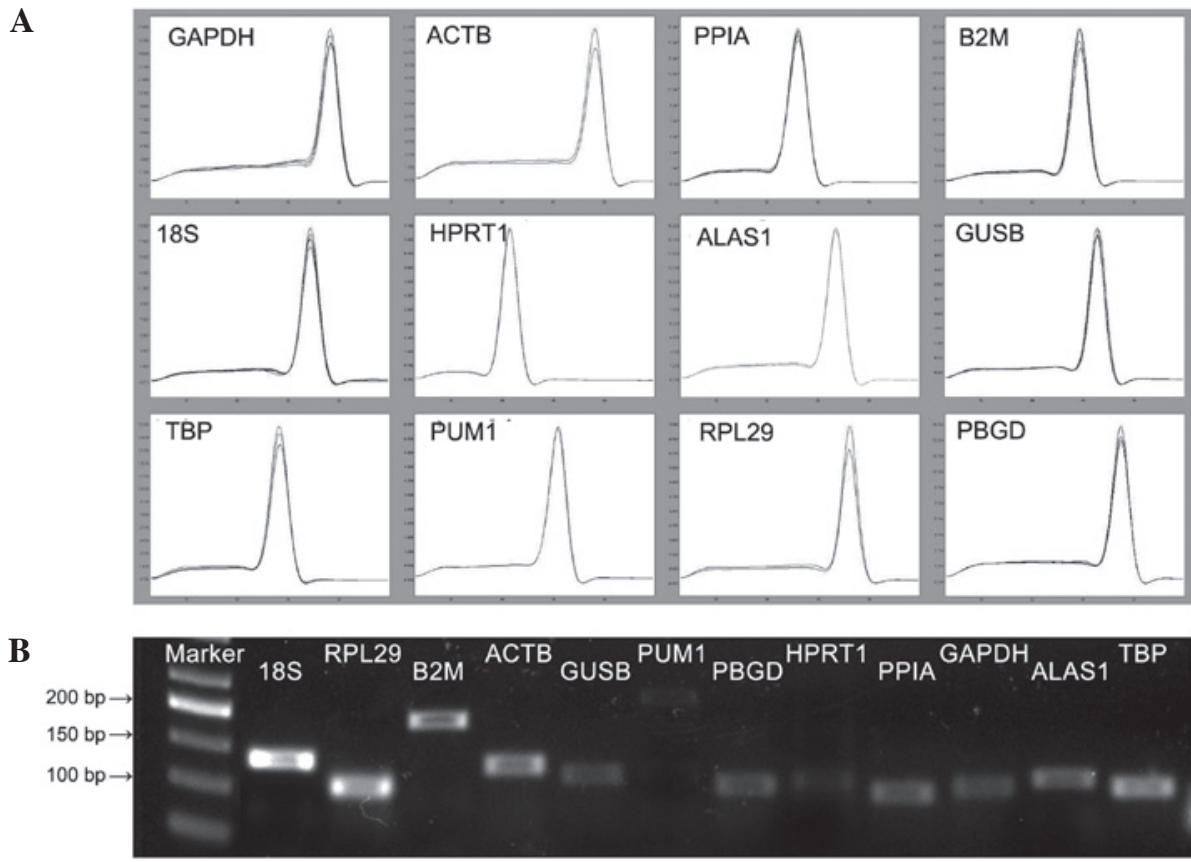

Figure 1. Specificity of RT-qPCR amplification. (A) Melting curves of RT-qPCR amplification products. (B) RT-qPCR amplification products were detected using 1\% agarose gel electrophoresis. RT-qPCR, reverse transcription-quantitative polymerase chain reaction; GAPDH, glyceraldehyde 3-phosphate dehydrogenase; ACTB, actin- $\beta$; PPIA, peptidylprolyl isomerase A; B2M, $\beta$-2-microglobulin; $18 \mathrm{~S}, 18 \mathrm{~S}$ ribosomal RNA; HPRT1, hypoxanthine phosphoribosyltransferase 1; ALAS1, 5 '-aminolevulinate synthase 1; GUSB, glucuronidase- $\beta$; TBP, TATA-box binding protein; PUM1, pumilio RNA binding family member 1; RPL29, ribosomal protein L29; PBGD, porphobilinogen deaminase.
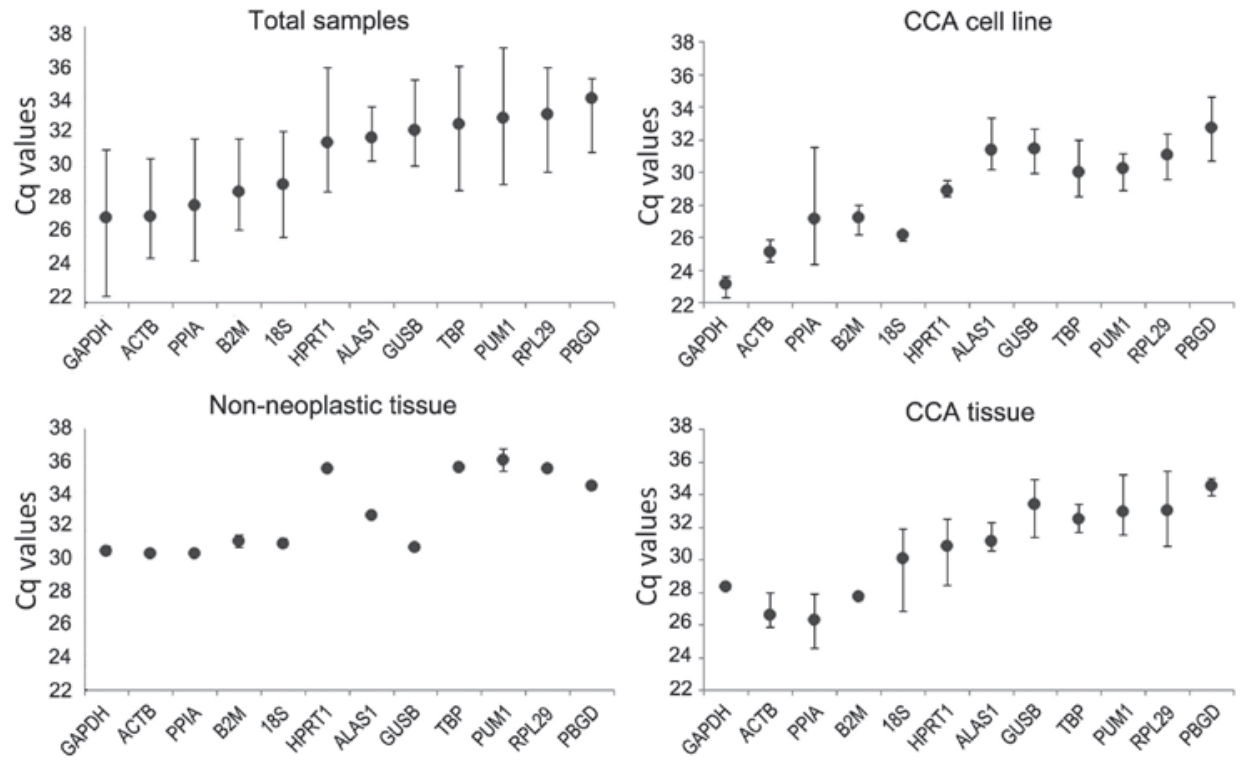

Figure 2. Mean Cq values of reference genes in various samples: Total samples group, CCA cell line, adjacent non-neoplastic tissue group and CCA tissue group. Whiskers represent the ranges for 20 matched samples. The results are presented as the mean \pm standard deviation. Cq, quantification cycle; CCA, cholangiocarcinoma; GAPDH, glyceraldehyde 3 -phosphate dehydrogenase; ACTB, actin- $\beta$; PPIA, peptidylprolyl isomerase A; B2M, $\beta$-2-microglobulin; $18 \mathrm{~S}$, 18 S ribosomal RNA; HPRT1, hypoxanthine phosphoribosyltransferase 1; ALAS1, 5'-aminolevulinate synthase 1; GUSB, glucuronidase- $\beta$; TBP, TATA-box binding protein; PUM1, pumilio RNA binding family member 1; RPL29, ribosomal protein L29; PBGD, porphobilinogen deaminase.

smallest $\mathrm{Cq}$ value and porphobilinogen deaminase (PBGD; also known as hydroxymethylbilane synthase) had the largest. There was a significant difference in the expression level of the candidate reference genes between the CCA tissues and the paired normal tissues $(\mathrm{Cq}, 24.12 \pm 0.38$ vs. $26.6 \pm 0.16$ in CAA vs. non-neoplasyic tissues, respectively; $\mathrm{P}=0.003$ ). The alteration in the $\mathrm{Cq}$ value of each group of candidate genes indicated that the expression level of the genes may differ between various experimental conditions.

Stability analysis of the candidate reference genes using GeNorm analysis. GeNorm was used to select the optimal reference genes. Two parameters were considered to quantify reference gene stability: M-value (average expression stability) 
A
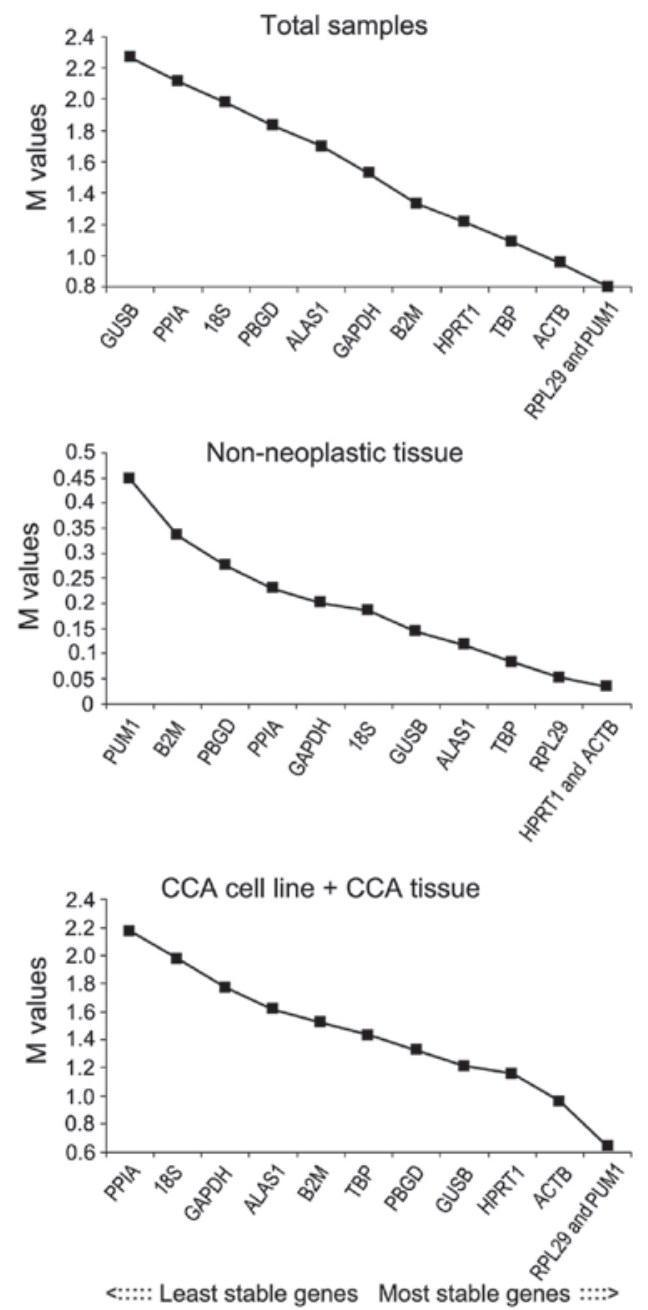
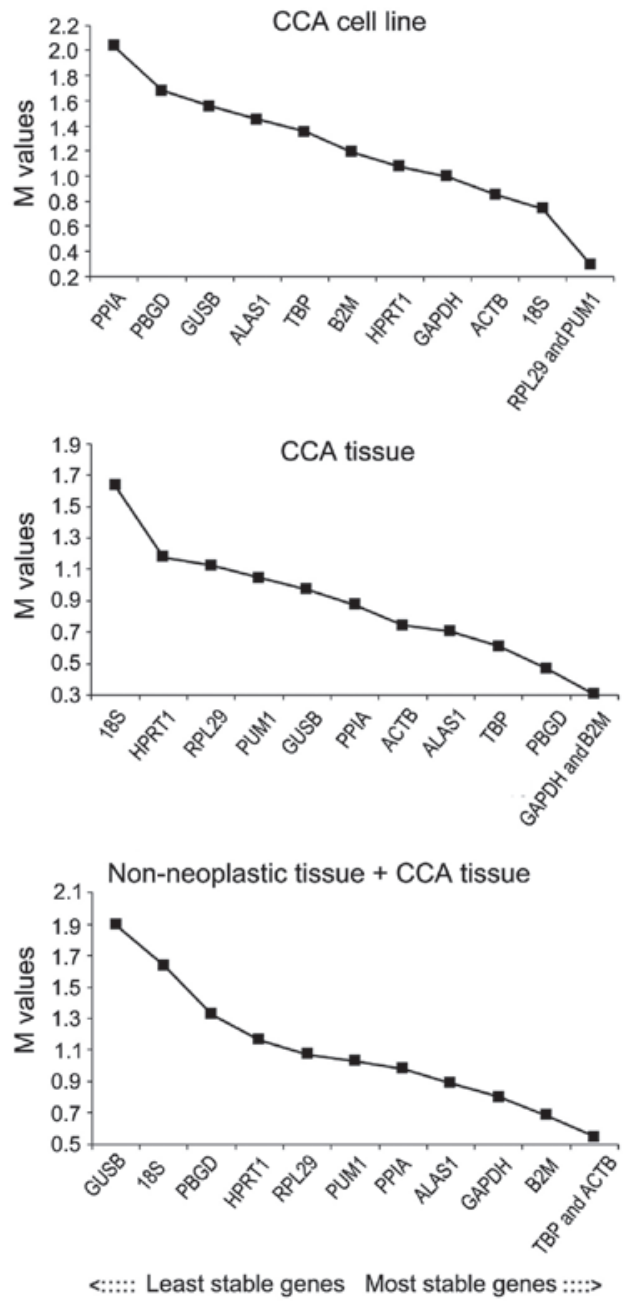

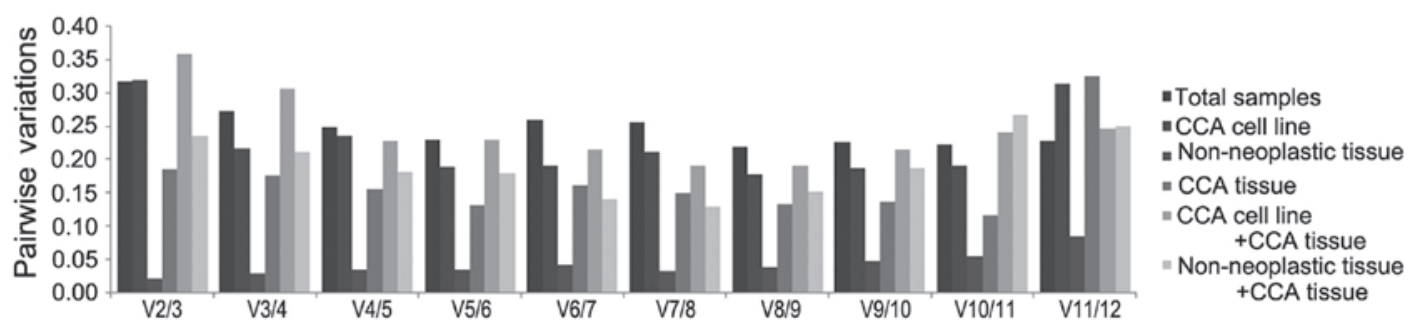

Figure 3. GeNorm analysis of 12 candidate reference genes. Results are presented according to the output file of the GeNorm program. (A) Stepwise exclusion of the least stable genes calculating the average expression stability as measured by the M-value in various samples. The $\mathrm{x}$-axis from left to right indicates the ranking of the reference genes according to expression stability and the y-axis indicates the stability as measured by the M-value. (B) Determination of the optimal number of reference genes for normalization based on the geNorm algorithm. $V$ value defines the pairwise variation between two sequential normalization. V, variable; CCA, cholangiocarcinoma; GAPDH, glyceraldehyde 3-phosphate dehydrogenase; ACTB, actin- $\beta$; PPIA, peptidylprolyl isomerase A; B2M, $\beta$-2-microglobulin; 18S, 18S ribosomal RNA; HPRT1, hypoxanthine phosphoribosyltransferase 1; ALAS1, 5'-aminolevulinate synthase 1; GUSB, glucuronidase- $\beta$; TBP, TATA-box binding protein; PUM1, pumilio RNA binding family member 1; RPL29, ribosomal protein L29; PBGD, porphobilinogen deaminase.

and $\mathrm{V}_{\mathrm{n}} / \mathrm{V}_{\mathrm{n}+1}$ (pair-wise variation). GeNorm software eliminates the gene with the highest $\mathrm{M}$-value and repeats the process until only two genes remain. The M-values of the 12 candidate reference genes in each group are shown in Fig. 3. The software analysis indicated that pumilio RNA binding family member 1 (PUM1) and ribosomal protein L29 (RPL29) were the most stable reference genes of the total samples group and the CCA cell line group. Similarly, in the CCA tissue and cell line group, PUM1 and RPL29 were the most stable, followed by actin- $\beta$ (ACTB). In the adjacent non-neoplastic group, ACTB and hypoxanthine phosphoribosyltransferase 1 (HPRT1) were the most stable. In CCA and adjacent non-neoplastic tissue groups, ACTB and TATA-box binding protein (TBP) were the most stable reference genes. In the CCA tissue group, the most stable reference genes were $\beta$-2-microglobulin (B2M) and GAPDH.

To determine the optimal number of required reference genes for each group, $\mathrm{V}_{\mathrm{n}} / \mathrm{V}_{\mathrm{n}+1}$ was evaluated using GeNorm; the default threshold value is 0.15 . However, as stated by Wan et al (20), 0.15 is not an absolute cutoff value, but rather an ideal value, which is dependent on the expression of the 
Total samples
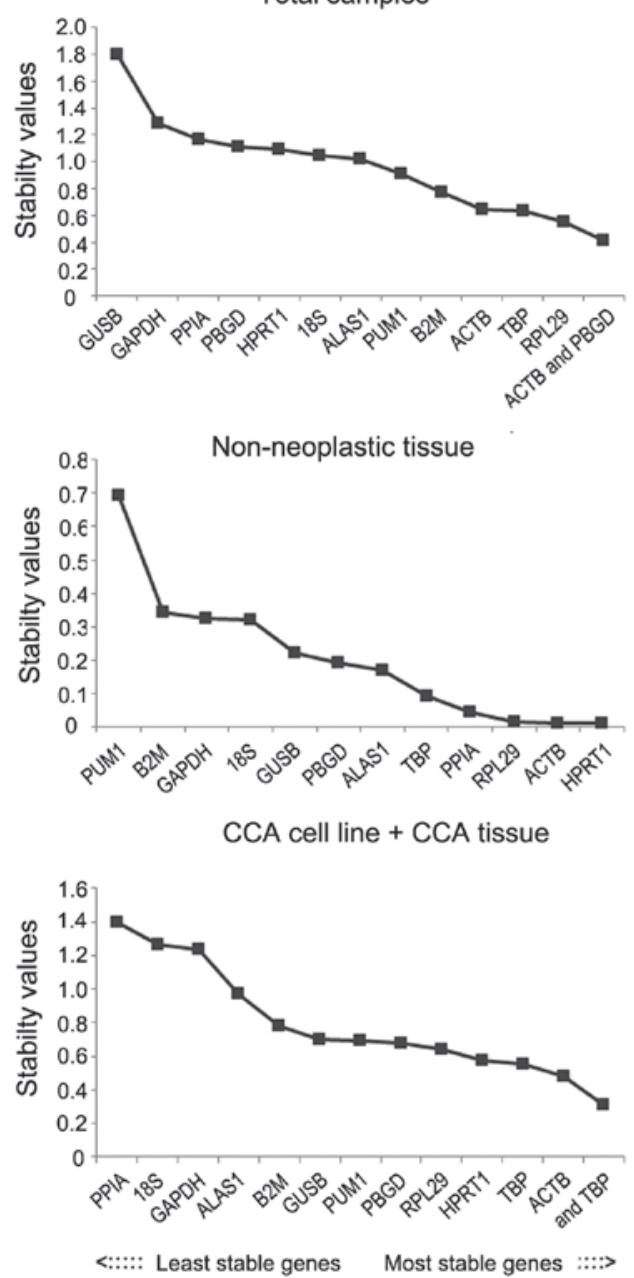

CCA cell line

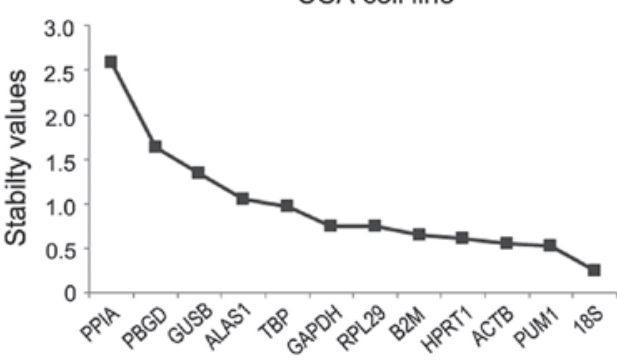

CCA tissue
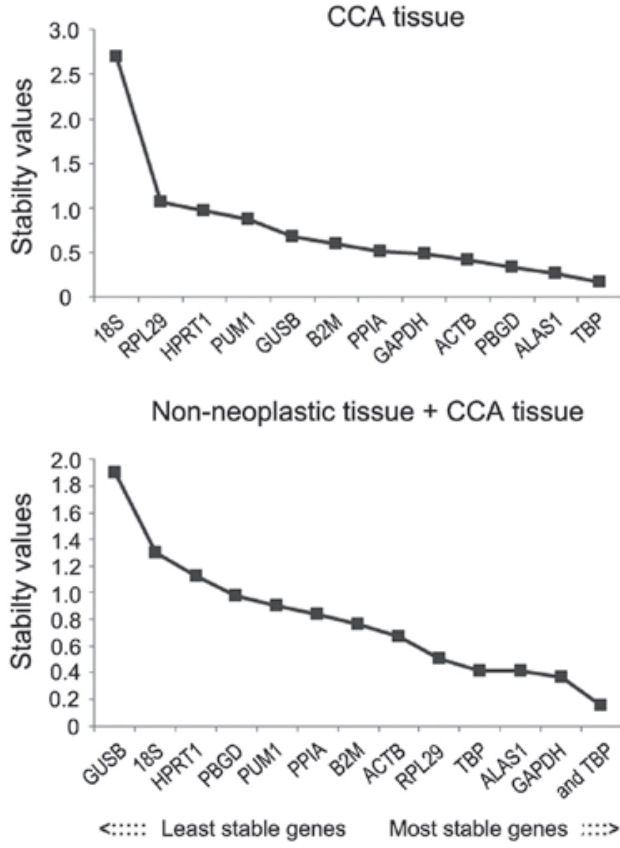

Figure 4. Candidate reference genes for normalization according to their expression stability calculated using NormFinder in various groups. The $\mathrm{x}$-axis from left to right represents the ranking of stability of the reference genes. CCA, cholangiocarcinoma; GAPDH, glyceraldehyde 3-phosphate dehydrogenase; ACTB, actin- $\beta$; PPIA, peptidylprolyl isomerase A; B2M, $\beta$-2-microglobulin; 18S, 18S ribosomal RNA; HPRT1, hypoxanthine phosphoribosyltransferase 1; ALAS1, 5 '-aminolevulinate synthase 1 ; GUSB, glucuronidase- $\beta$; TBP, TATA-box binding protein; PUM1, pumilio RNA binding family member 1 ; RPL29, ribosomal protein L29; PBGD, porphobilinogen deaminase.

genes and the diversity of the samples tested. The number of reference genes recommended to be used in each group is shown in Fig. 3B. The combination of 8 reference genes in the total sample group is the optimum $(\mathrm{V} 8 / 9=0.219)$, while 7 is the optimum in the CCA tissue group ( $\mathrm{V} 7 / 8=0.150)$; in addition, the adjacent non-neoplastic tissue group has an optimum combination of 11 reference genes (V11/12=0.107). The combination of 8 reference genes in the CCA cell line group is the optimum (V8/9=0.178), while 6 is the optimum in matched pairs of adjacent non-neoplastic and CCA tissues group (V6/7=0.140). The CCA tissue and cell line group has an optimum combination of 7 reference genes $(\mathrm{V} 7 / 8=0.191)$.

Stability analysis of candidate reference genes using NormFinder analysis. NormFinder was used to validate the most stable genes (Fig. 4). According to the analysis, in the total samples group, the most stable reference gene was RPL29, followed by TBP, and the recommended combination was ACTB and PBGD. In the CCA cell line group, the most stable reference gene was $18 \mathrm{~S}$ ribosomal RNA (18SrRNA), followed by PUM1. In the CCA cell line and CCA tissue group, ACTB was the most stable followed by TBP. In the adjacent non-neoplastic group, HPRT1 was the most stable gene, followed by ACTB. In CCA tissue and adjacent non-neoplastic tissue group, GAPDH was the most stable reference gene, followed by 5'-aminolevulinate synthase 1 (ALAS1). In CCA tissue group, the most stable reference gene was TBP followed by ALAS1.

Stability analysis of the candidate reference genes using BestKeeper analysis. BestKeeper also identified the most stably expressed genes by comparing the r-value and the SD as shown in Figs. 5 and 6. The number of candidate reference genes that could be analyzed by this software was limited (14); therefore, only 10 candidate reference genes were analyzed for each group, based on the result of GeNorm. The analysis demonstrated that the SD value in the total samples group was $>1$, which does not necessarily indicate that the 10 candidate internal reference genes are all unstable, as results from a single software program is not sufficient to confirm this. According to the r-value, the most stable internal reference gene in the total samples group was ACTB. In the CCA cell line group, the most stable reference gene was 18SrRNA, followed by PUM1. In the CCA cell line and tissue group, ACTB was the most stable reference gene followed by B2M. 

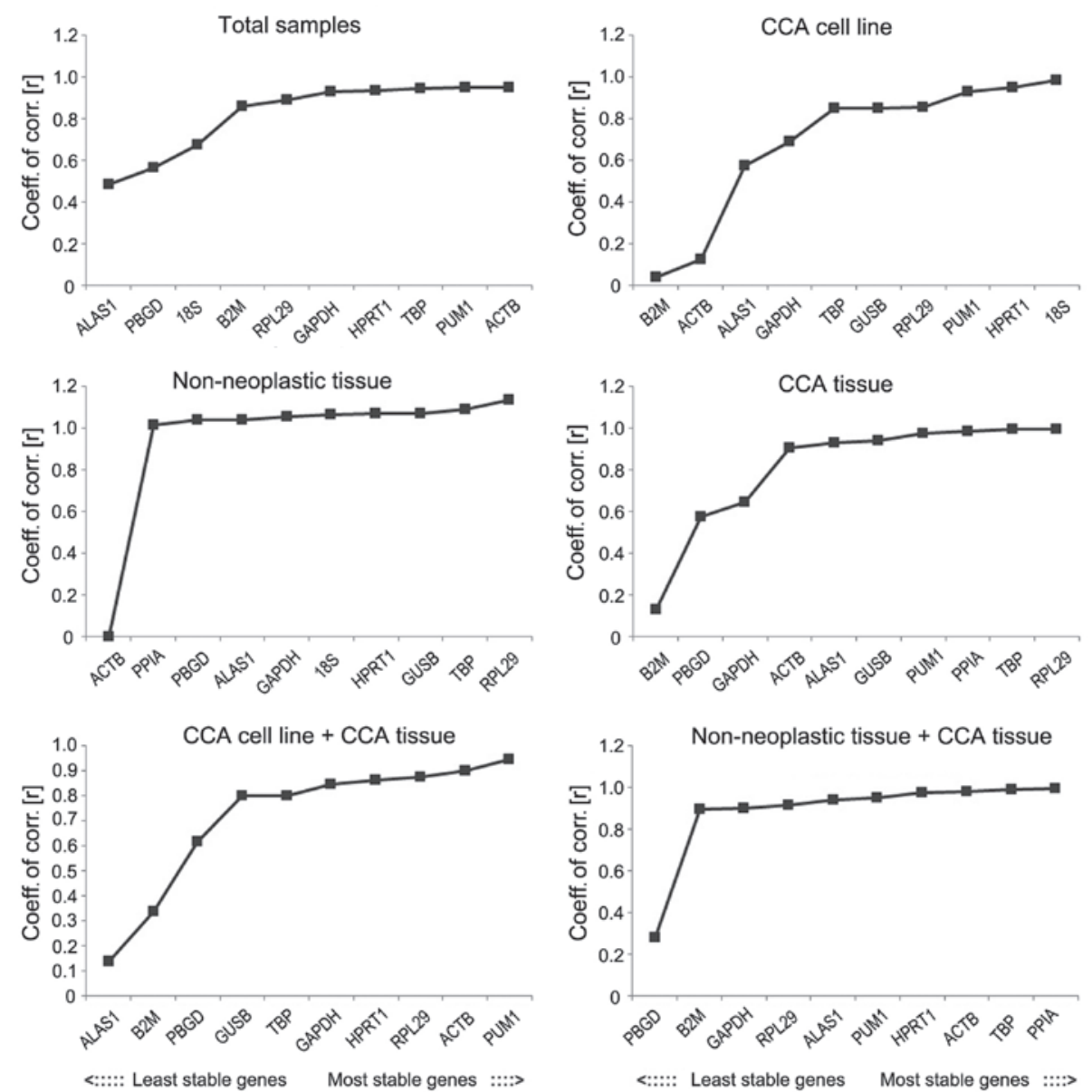

Figure 5. Stability values of the candidate reference genes evaluated using BestKeeper in various groups, which shows the r-values of the candidate reference genes; the higher the r-value, the more stability the reference gene possesses. CCA, cholangiocarcinoma; r-value, coefficient of correlation; GAPDH, glyceraldehyde 3-phosphate dehydrogenase; ACTB, actin- $\beta$; PPIA, peptidylprolyl isomerase A; B2M, $\beta$-2-microglobulin; $18 \mathrm{~S}, 18 \mathrm{~S}$ ribosomal RNA; HPRT1, hypoxanthine phosphoribosyltransferase 1; ALAS1, 5'-aminolevulinate synthase 1; GUSB, glucuronidase- $\beta$; TBP, TATA-box binding protein; PUM1, pumilio RNA binding family member 1; RPL29, ribosomal protein L29; PBGD, porphobilinogen deaminase.

In the adjacent non-neoplastic group, RPL29 was the most stable gene, followed by TBP. In CCA tissue and adjacent non-neoplastic tissue group, ALAS1 was the most stable reference gene, followed by PBGD. In CCA tissue group, the most stable reference gene was TBP, followed by ALAS1.

Relative KRAS expression. The analysis of a target gene's relative expression is affected by the selection of reference genes (7). As shown in Fig. 7, when TBP, GAPDH, ACTB and ALAS1, which are recommended by the present study, were used as reference genes for KRAS (a target gene), there was no significant difference in the expression of the KRAS gene. The level of expression of KRAS in CCA tissue did not vary when these samples were normalized using TBP $(\mathrm{P}=0.161)$, GAPDH $(\mathrm{P}=0.156)$, ACTB $(\mathrm{P}=0.128)$ or ALAS1 $(\mathrm{P}=0.157)$. However, when glucuronidase- $\beta$ (GUSB; $P=0.004$ ) was selected as the reference gene for normalization, the relative expression of KRAS in CCA tissue was clearly different, compared to the other candidate reference genes.

\section{Discussion}

Since RT-qPCR is fast, has a high sensitivity and provides quantifiable data, it is commonly used for the analysis of gene expression (17). However, during the RT-qPCR process, the difference in quality and quantity of RNA and synthetic efficiency of cDNA and PCR amplification may lead to significant deviations of the results. Therefore, during the detection of target gene expression, a gene with a stable expression is required as a reference. Recent studies have revealed defects in all commonly used reference genes, which often vary greatly in expression quantity in various types of cells and tissues, stages of cell amplification and organ development and in vitro culture and experimental conditions $(9,10,20-22)$. To the best of our knowledge, there have been no systematic comparisons performed concerning the stability of commonly-used reference genes in CCA tissues and cell lines. Early diagnosis of CCA is challenging, and it is problematic to achieve a satisfactory therapeutic effect in patients $(2,3)$. In addition, evaluating the prognosis of patients with CCA is inaccurate. Therefore, with the development of CCA-associated studies using gene profiling, it is essential to determine stable and reliable reference genes.

In order to acquire accurate experimental data and reliable conclusions, the present study was designed with following features: Matching of malignant and non-malignant specimens from the same patient was adopted so as to reduce individual differences; the specimens were not collected according to the 

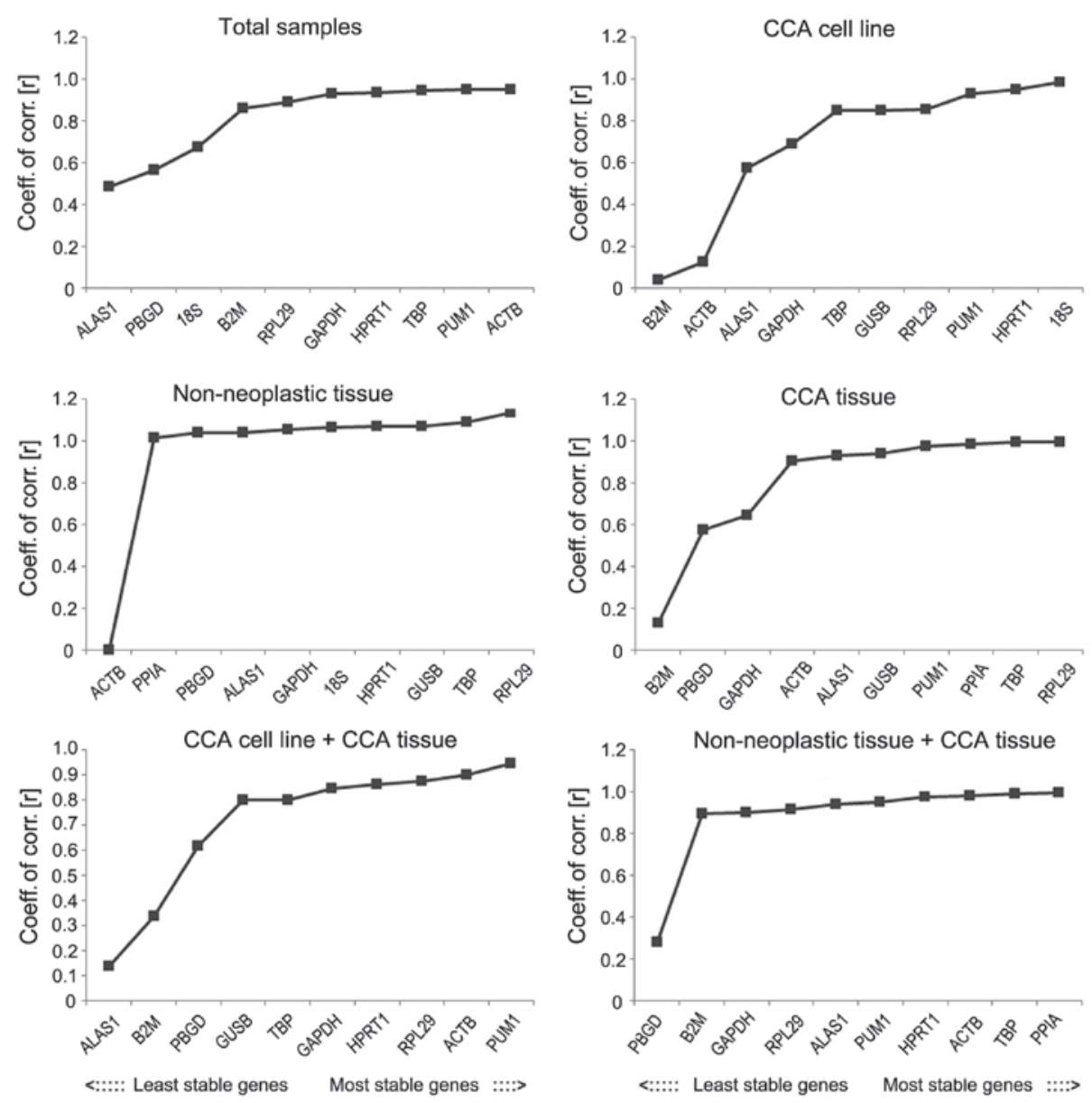

Figure 6. Stability values of the candidate reference genes evaluated using BestKeeper in various groups, which shows standard deviation values of the candidate reference genes. CCA, cholangiocarcinoma; GAPDH, glyceraldehyde 3-phosphate dehydrogenase; ACTB, actin- $\beta$; PPIA, peptidylprolyl isomerase A; B2M, $\beta$-2-microglobulin; 18S, 18S ribosomal RNA; HPRT1, hypoxanthine phosphoribosyltransferase 1; ALAS1, 5'-aminolevulinate synthase 1; GUSB, glucuronidase- $\beta$; TBP, TATA-box binding protein; PUM1, pumilio RNA binding family member 1 ; RPL29, ribosomal protein L29; PBGD, porphobilinogen deaminase.

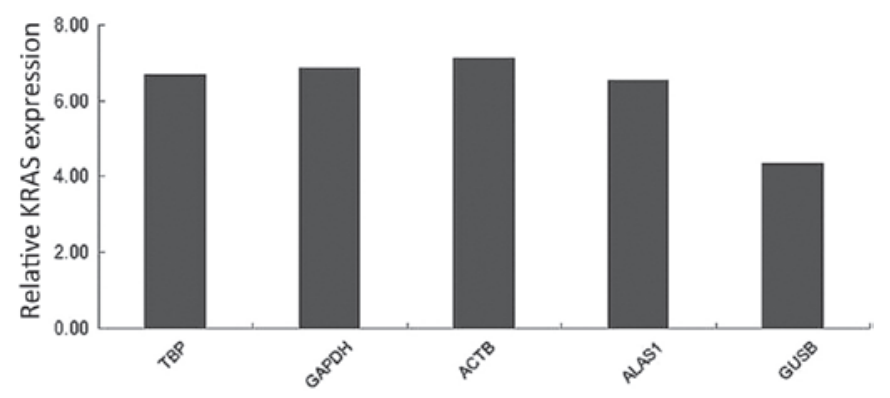

Figure 7. Relative quantification of KRAS, as normalized by TBP, GAPDH, ACTB, ALAS1 and GUSB in 20 matched pairs of adjacent non-neoplastic and cholangiocarcinoma tissues. KRAS, Kirsten rat sarcoma viral oncogene homolog; TBP, TATA-box binding protein; GAPDH, glyceraldehyde 3 -phosphate dehydrogenase; ACTB, actin- $\beta$; ALAS1, 5'-aminolevulinate synthase 1 ; GUSB, glucuronidase- $\beta$.

grade and stage of tumors, and as previous studies indicate, the expression of a reference gene is not directly associated with the grade and stage of malignant tumors (21); the selected samples were all characterized as malignant tissue samples by the pathology department of the China-Japan Union Hospital of Jilin University, and the pathology observed in the samples indicated the most common pathological type of CCA, adenocarcinoma; the QBC939 cell line was also used as it is a commonly used human CCA cell line; the stability evaluation on reference genes for the selected histological samples and cell lines of human CCA was implemented to make the experimental results more comprehensive, compared with previous studies $(9,10,21,22)$ where the stability evaluation on reference genes was restricted on ceratin tissues or cell lines; and the stability of 12 common reference genes was compared.

The present study utilized GeNorm to analyze the reference genes. This software is based on a pairwise-comparison statistical model. By calculating the $\mathrm{M}$ - and V-values, the present study determined the two most stable reference genes and the best reference gene combination for various tissue groups and the CCA cell line. By calculating the value of $\mathrm{V}$, the optimal reference genes combination number for each group was greater than six. Therefore, considering a previous study (8), the present study recommends three internal genes as the most appropriate. NormFinder, which uses analysis of variance as the statistical model, demonstrated similar results to those observed with GeNorm. Finally, in order to reduce the one-sidedness of GeNorm and NormFinder, the present study also used BestKeeper for analysis. However, the analysis results of BestKeeper are different to those observed with GeNorm and NormFinder. Previous studies hypothesize that 
this may be due to differences in the statistical model that BestKeeper uses; therefore, it is less effective for ranking reference gene stability compared with GeNorm and NormFinder (20-22). Based on comparisons of results among the three programs, the present study recommends the following most stable reference genes, which have been confirmed through analysis by $\geq 2$ programs: Total samples group, RPL29; CCA tissue group, TBP; CCA cell line and CCA tissue group, ACTB; CCA cell line group, 18SrRNA; CCA tissue and adjacent non-neoplastic tissue group, ALAS1; and adjacent non-neoplastic tissue group, HPRT1.

The observation that KRAS gene expression depended on the normalization method used illustrates the significance of reference genes for obtaining dependable expression data. If RAS genes mutate, the encoded $\mathrm{P} 21$ protein conformation may alter, leading to excessive cell proliferation and eventually causing the development of cancer (23). The RAS gene most associated with CCA is KRAS (23), followed by NRAS. The present study selected the most stable reference genes recommended by GeNorm, NormFinder and BestKeeper, and also GUSB, which has poor stability, as the standard for relative quantification analysis (8). The results indicated that the relative expression quantities of KRAS varied significantly, illustrating that a suitable reference gene is essential for gene profiling of CCA. Similar erroneous normalizations have been observed in other tissue types, including gastric cancer, when inadequate control genes or normalizing strategies are performed (24).

In conclusion, the present study recommends the most suitable reference genes and reference gene combinations for human CCA tissue and cell lines to aid in performing gene expression profile analysis. A reliable normalization strategy in such studies may contribute to an improved understanding of the biology of these rare tumors. Elucidation of the molecular expression signatures may lead to more accurate diagnostics as well as identification of prognostic factors and, ultimately, targets for future therapeutics for patients with CCA.

\section{Acknowledgements}

The present study was supported by the Key Foundation of Jilin Provincial Science and Technology Department (grant nos., 20130727038YY and 20100942; Changchun, China) and Jilin Provincial Development and Reform Commission (grant no., 20101928; Changchun, China).

\section{References}

1. Lazaridis $\mathrm{KN}$ and Gores GJ: Cholangiocarcinoma Gastroenterology 128: 1655-1667, 2005.

2. Friman S: Cholangiocarcinoma - current treatment options. Scand J Surg 100: 30-34, 2011.

3. Cardinale V, Semeraro R, Torrice A, Gatto M, Napoli C, Bragazzi MC, Gentile R and Alvaro D: Intra-hepatic and extra-hepatic cholangiocarcinoma: New insight into epidemiology and risk factors. World J Gastrointest Oncol 2: 407-416, 2010.

4. Brown KM: Multidisciplinary approach to tumors of the pancreas and biliary tree. Surg Clin North Am 89: 115-131, 2009.

5. Bustin SA: Absolute quantification of mRNA using real-time reverse transcription polymerase chain reaction assays. J Mol Endocrinol 25: 169-193, 2000.
6. Bustin SA, Benes V, Nolan T and Pfaffl MW: Quantitative real-time RT-PCR-a perspective. J Mol Endocrinol 34: 597-601, 2005.

7. Derveaux S, Vandesompele J and Hellemans J: How to do successful gene expression analysis using real-time PCR. Methods 50: 227-230, 2010.

8. Vandesompele J, De Preter K, Pattyn F, Poppe B, Van Roy N, De Paepe A and Speleman F: Accurate normalization of real-time quantitative RT-PCR data by geometric averaging of multiple internal control genes. Genome Biol 3: RESEARCH0034, 2002

9. de Jonge HJ, Fehrmann RS, de Bont ES, Hofstra RM, Gerbens F, Kamps WA, de Vries EG, van der Zee AG, te Meerman GJ and ter Elst A: Evidence based selection of housekeeping genes. PLoS One 2: e898, 2007.

10. Ho-Pun-Cheung A, Bascoul-Mollevi C, Assenat E, Bibeau F, Boissière-Michot F, Cellier D, Ychou M and Lopez-Crapez E: Validation of an appropriate reference gene for normalization of reverse transcription-quantitative polymerase chain reaction data from rectal cancer biopsies. Anal Biochem 388: 348-350, 2009.

11. Bustin SA and Mueller R: Real-time reverse transcription PCR (qRT-PCR) and its potential use in clinical diagnosis. Clin Sci (Lond) 109: 365-379, 2005.

12. Hruz T, Wyss M, Docquier M, Pfaffl MW, Masanetz S, Borghi L, Verbrugghe P, Kalaydjieva L, Bleuler S, Laule O, et al: RefGenes: Identification of reliable and condition specific reference genes for RT-qPCR data normalization. BMC Genomics 12: 156, 2011.

13. Kimmitt PT, Tabrizi SN, Crosatti M, Garland SM, Schober PC, Rajakumar K and Chapman CA: Pilot study of the utility and acceptability of tampon sampling for the diagnosis of Neisseria gonorrhoeae and Chlamydia trachomatis infections by duplex realtime polymerase chain reaction in United Kingdom sex workers. Int J STD AIDS 21: 279-282, 2010.

14. Pfaffl MW, Tichopad A, Prgomet C and Neuvians TP: Determination of stable housekeeping genes, differentially regulated target genes and sample integrity: BestKeeper-Excel-based tool using pair-wise correlations. Biotechnol Lett 26: 509-515, 2004.

15. Tannapfel A, Benicke M, Katalinic A, Uhlmann D, Köckerling F, Hauss J and Wittekind C: Frequency of p16(INK4A) alterations and K-ras mutations in intrahepatic cholangiocarcinoma of the liver. Gut 47: 721-727, 2000.

16. Edge SB and Compton CC: The American Joint Committee on Cancer: The 7th edition of the AJCC cancer staging manual and the future of TNM. Ann Surg Oncol 17: 1471-1474, 2010.

17. Livak KJ and Schmittgen TD: Analysis of relative gene expression data using real-time quantitative PCR and the 2(-Delta Delta C(T)) Method. Methods 25: 402-408, 2001.

18. Ginzinger DG: Gene quantification using real-time quantitative PCR: An emerging technology hits the mainstream. Exp Hematol 30: 503-512, 2002.

19. Livak KJ and Schmittgen TD: Analysis of relative gene expression data using real-time quantitative PCR and the 2(-Delta Delta C(T)) Method. Methods 25: 402-408, 2001.

20. Wan H, Zhao Z, Qian C, Sui Y, Malik AA and Chen J: Selection of appropriate reference genes for gene expression studies by quantitative real-time polymerase chain reaction in cucumber. Anal Biochem 399: 257-261, 2010.

21. Ohl F, Jung M, Xu C, Stephan C, Rabien A, Burkhardt M, Nitsche A, Kristiansen G, Loening SA, Radonić A and Jung K: Gene expression studies in prostate cancer tissue: Which reference gene should be selected for normalization? J Mol Med (Berl) 83: 1014-1024, 2005.

22. Liu S, Zhu P, Zhang L, Ding S, Zheng S, Wang Y and Lu F: Selection of reference genes for RT-qPCR analysis in tumor tissues from male hepatocellular carcinoma patients with hepatitis B infection and cirrhosis. Cancer Biomark 13: 345-349, 2013

23. Isa T, Tomita S, Nakachi A, Miyazato H, Shimoji H, Kusano T, Muto Y and Furukawa M: Analysis of microsatellite instability, K-ras gene mutation and p53 protein overexpression in intrahepatic cholangiocarcinoma. Hepatogastroenterology 49: 604-608, 2002.

24. Kumar V, Sharma R, Trivedi PC, Vyas GK and Khandelwal V: Traditional and novel references towards systematic normalization of qRT-PCR data in plants. Australian J Crop Sci 5: 1455-1468, 2011. 